\title{
Two new species of Olecryptotendipes Zorina, 2007 from China (Diptera, Chironomidae)
}

\author{
Chun-Cai Yan ${ }^{1, \dagger}$, Xin-Hua Wang ${ }^{2, \ddagger}$, Wen-Jun Bu ${ }^{2, \S}$ \\ I Tianjin Key Laboratory of Cyto-Genetical and Molecular Regulation, College of Life Sciences, Tianjin Nor- \\ mal University, Tianjin 300387, China; 2 College of Life Sciences, Nankai University, Tianjin 300071, China \\ † urn:lsid:zoobank.org:author:BAEAE628-EE37-44C9-9FD5-840BC3F9B838 \\ ‡ urn:lsid:zoobank.org:author:ACC3483B-5DD1-4F2D-88F7-1CE4C6458B68 \\ § urn:lsid:zoobank.org:author:072B7D13-17B4-4C11-84D6-08E7168176C8 \\ Corresponding author: Chun-Cai Yan (flyfish113@163.com)
}

Academic editor: Torsten Dikow | Received 26 April 2012 | Accepted 4 July 2012 | Published 17 July 2012 urn:lsid:zoobank.org:pub:F18C2EB8-D811-4E31-BEB2-3B15AB85F15D

Citation: Yan C-C, Wang X-H, Bu W-J (2012) Two new species of Olecryptotendipes Zorina, 2007 from China (Diptera, Chironomidae). ZooKeys 208: 41-49. doi: 10.3897/zookeys.208.3299

\section{Abstract}

Two new species, Olecryptotendipes exilis sp. n. and O. melasmus sp.n. are described and illustrated as males and Chinese males of $O$. lenzi are re-examined. A key to all known males of Olecryptotendipes is provided.

\section{Keywords}

Chironomidae, Olecryptotendipes, new species, key, China

\section{Introduction}

Based on the morphology of the males in the Russian Far East, Cryptotendipes lenzi Zorina, 2001 and C. secundus Zorina, 2003 were described by Zorina (2001, 2003). Zorina (2007) collected the larvae and pupae of C. lenzi, and showed the two species should be placed in a new genus, named Olecryptotendipes Zorina, 2007. The type species is Cryptotendipes lenzi Zorina, 2001. To date, the genus only contains the two aforementioned species. Yan et al. (2005) recorded Cryptotendipes lenzi from Xinjiang Autonomous Region in China. 
The males of Olecryptotendipes are characterized by Y-shaped anal tergite bands; posterior part of tergite IX elongated with setae; superior volsella with sclerotized part and membranous ridge, with dorsal and ventral setae, microtrichia absent or present ventrally and weak inferior volsella (Zorina 2007). For the diagnosis of pupa and larva, refer to Zorina (2007).

In the present paper, two new species are recorded. Prof. Ole Sxther and Dr. M. Spies have checked the specimens. The two new species don't belong to the genera Cryptotendipes Lenz, 1941 and Chernouskiia Sæther, 1977 because of the presence of sclerotized superior volsella and lobate inferior volsella. In addition, the species of the genus Cryptotendipes lack an inferior volsella and the margin of the gonostylus is usually with an expansion. Species of the genus Chernovskiia are also without lobate inferior volsella, but with foot-shaped superior volsella, which also present in species of genera Paracladopelma Harnisch, 1923 and Beckidia Kieffer, 1913. The Y-shaped anal tergite bands (Figs 2, 8), the shoulder-like margin of tergite IX (Figs 2, 8), the sclerotized superior volsella (Figs 4, 10) and lobate inferior volsella (Figs 3, 9) show them to belong to the genus Olecryptotendipes (O. Sæther and M. Spies, pers. comm.). However, we have no specimens of larvae and pupae which are important to place the species properly.

The larvae of Olecryptotendipes inhabit sandy substrate in rivers (Zorina 2007). The Chinese specimens were collected from temperate zones and subtropical mountain areas in Palaearctic and Oriental China (Map 1).

Two new species, $O$. exilis sp. n. and $O$. melasmus sp. n., are described and illustrated based on material from China and a key to the males of Olecryptotendipes is provided.

\section{Material and methods}

The terminology follows Sæther (1980) with the additions and corrections given by Sæther (1990). The material examined was mounted on slides in Canada balsam following the procedure outlined by Sæther (1969).

The type material and other material studied are housed in the College of Life Science, Department of Biology, Nankai University, Tianjin, China (BDN).

\section{Taxonomy}

\section{Amended generic diagnosis}

Based on Zorina (2007) and the Chinese specimens, we amend the generic diagnosis. The following combination of characters will separate the genus Olecryptotendipes from other members of the Harnischia complex: Total length 2.7-3.6 mm; AR 1.83-2.24; frontal tubercles absent; anal tergite bands of Y-shaped; posterior margin of tergite IX 


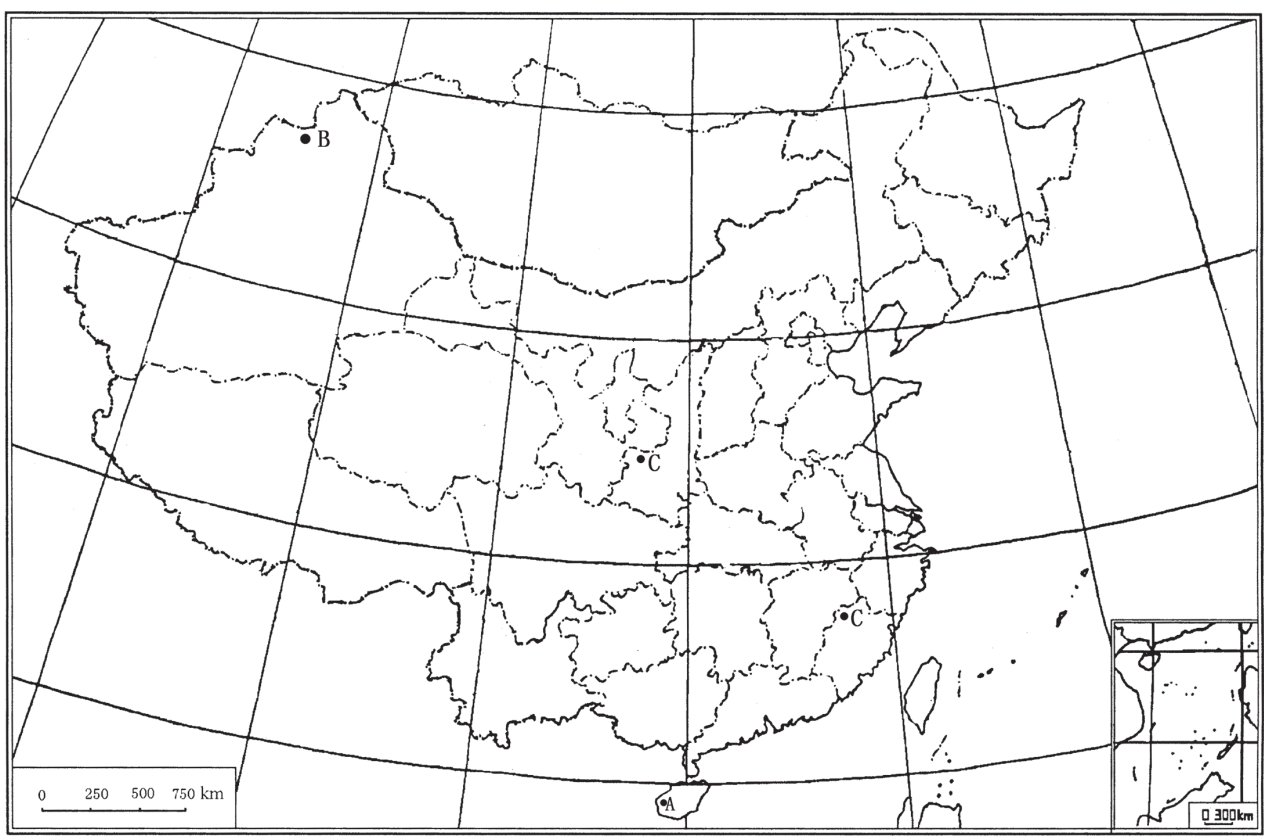

Map I. Distribution in China for the genus Olecryptotendipes. A O. exilis sp. n. B O. lenzi Zorina C O. melasmus sp. $\mathrm{n}$.

with caudolateral shoulders; anal point parallel-sided or widest at about apical 1/3; superior volsella consisting of sclerotized part, dorsal and ventral setae present; inferior volsella with a weak blunt caudal projection, covered with microtrichia; gonostylus parallel-sided or slender to apex, inner margin without expansion.

\section{Olecryptotendipes exilis sp. $\mathbf{n}$.}

urn:Isid:zoobank.org:act:843E24C2-A4C2-4BDF-B681-582F6CBF1692

http://species-id.net/wiki/Olecryptotendipes_exilis

Figs $1-4$

Diagnostic characters. The species is separated by the slender posterolateral projection of the superior volsella and the lobate inferior volsella, the posterolateral weak lobes of the anal tergite, and the parallel-sided anal point.

Description. Male imago $(\mathrm{n}=2)$. Total length $2.70-2.94 \mathrm{~mm}$. Wing length $1.38-1.50 \mathrm{~mm}$. Total length / wing length 1.96. Wing length / length of profemur $1.92-2.08$.

Coloration. Thorax and legs dark brown. Abdomen with tergite I-V yellowish brown and tergite VI-VIII and hypopygium dark brown.

Head. AR 1.97-2.02. Ultimate flagellomere 590-600 mm long. Frontal tubercles absent. Temporal 13-15 setae, including 4 inner verticals, 5-6 outer verticals and 4-5 

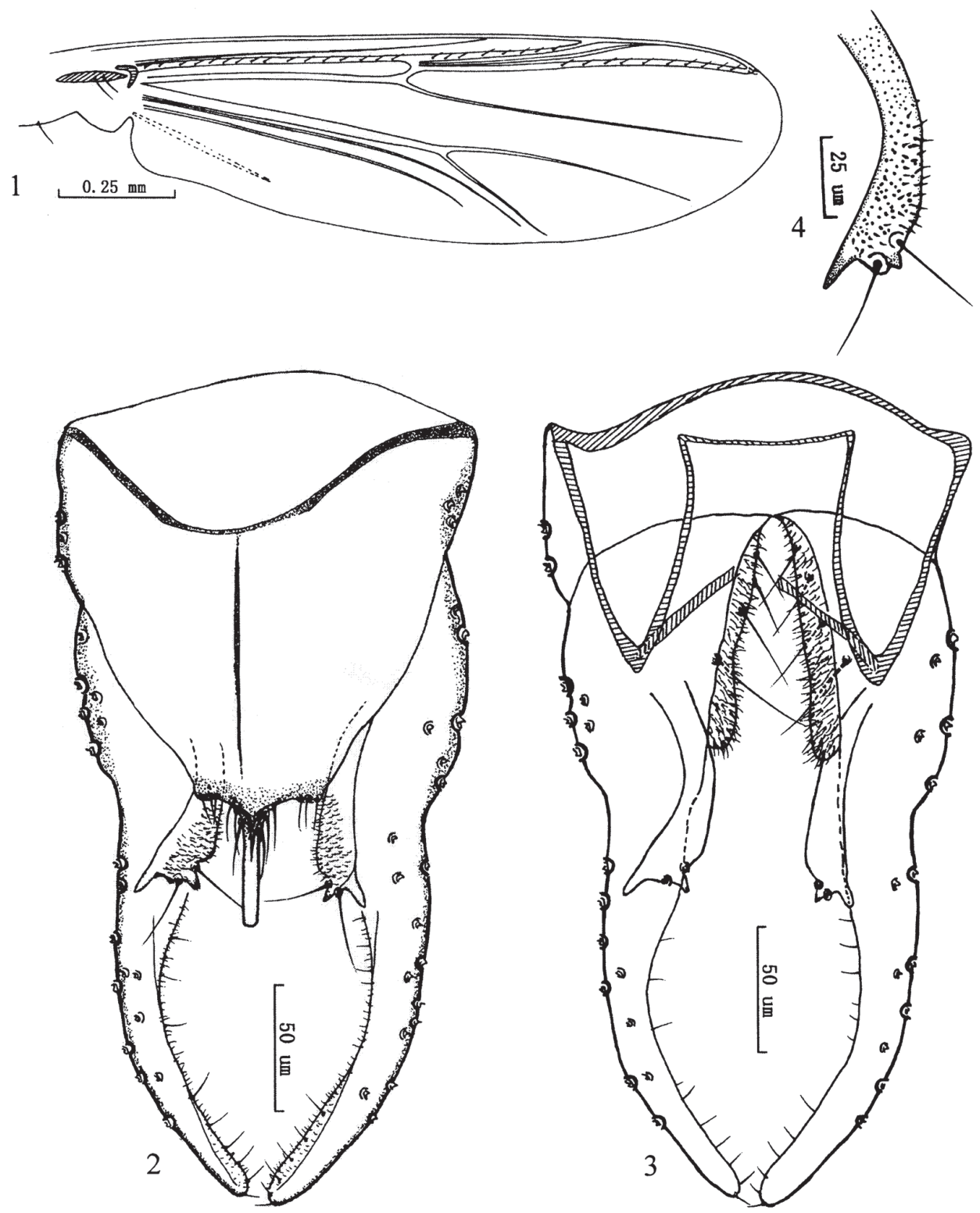

Figures I-4. Olecryptotendipes exilis sp. n., male. I wing. $\mathbf{2}$ hypopygium (dorsal view ) $\mathbf{3}$ hypopygium (ventral view) $\mathbf{4}$ superior volsella.

postorbitals. Clypeus with 13 setae. Tentorium 103-110 mm long, 28-30 mm wide. Palpomere lengths (in mm): 36-38, 38-40, 118-120, 140-145, 203-210. Palp segment $5^{\text {th }} / 3^{\text {rd }} 1.72-1.75$.

Thorax. Antepronotals with 6-7 setae, dorsocentrals 8-10, acrostichals 8, prealars 3. Scutellum with $12-13$ setae. 
Wing (Fig. 1). VR 1.19-120, $\mathrm{R}$ with $16-18$ setae, $\mathrm{R}_{1}$ with $8-10$ setae, $\mathrm{R}_{4+5}$ with 10-11 setae. Brachiolum 2 setae. Squama with 2-3 setae.

Legs. Front tibia with 3 subapical setae, 113-120, 138-144 and 141-150 $\mu \mathrm{m}$ long, spurs of mid tibia 30-35 and 37-46 $\mu \mathrm{m}$ long excluding comb, comb with 32-36 teeth, $10 \mu \mathrm{m}$ long; spurs of hind tibia $28-30$ and 38-47 $\mu \mathrm{m}$ long excluding comb, comb with 42-48 teeth, $10-12 \mu \mathrm{m}$ long. Ta ${ }_{1}$ of mid legs with only 1 sensilla chaetica, sensilla chaetica absent in hind legs. Lengths (in $\mu \mathrm{m}$ ) and proportions of legs as in Table 1.

Hypopygium (Figs 2-4). Tergite IX with weak lobes bearing 3-4 setae at each side of base of anal point. Laterosternite IX with 3 setae. Anal point 45-50 mm long, 5-6 mm wide, originating from caudal margin of anal tergite, completely parallel-side. Anal tergite bands Y-shaped. Phallapodeme 75-82 mm long. Transverse sternapodeme 56-60 mm long. Superior volsella (Fig. 4) slightly curved, with apical, partially sclerotized beak-like protrusion and slender spur-like posterolateral projection, bearing two long setae beside the beak-like protrusion, and covered with microtrichia in inner parts. Inferior volsella with a moderately blunt caudal projection, covered with microtrichia, and not reaching beyond margin of anal tergite. Gonocoxite 98-104 mm long, with 4 strong inner marginal setae. Gonostylus 168-175 mm long, slightly swollen at base, curved medially, moderately slender to apex, bearing 17-20 setae along inner margin. HR 0.58-0.59; HV 1.61-1.68.

Type material. Holotype $\partial$ (BDN No. 1291). CHINA: Hainan Province, Ledong Li Nationality Autonomous County, Jianfengling Nature Conservation area, $18^{\circ} 14^{\prime} 45.96 " \mathrm{~N}, 109^{\circ} 30^{\prime} 42.69^{\prime} \mathrm{E}, 21 . i v .1985$, X. Wang. Paratype $1{ }^{\Uparrow}$ (BDN No. 03578), same data as holotype.

Etymology. From Latin exilis, slender, in reference to the slender posterolateral projections of superior volsella.

Distribution. The species was collected in a subtropical mountain area in Hainan province in Oriental China.

Table I. Lengths $(\mu \mathrm{m})$ and proportion of legs of Olecryptotendipes exilis sp. n., male $(\mathrm{n}=2)$.

\begin{tabular}{c|c|c|c|c|c|c|c|c}
\hline & $\mathbf{F e}$ & $\mathbf{t i}$ & $\mathbf{t a}_{\mathbf{1}}$ & $\mathbf{t a}_{\mathbf{2}}$ & $\mathbf{t a}_{\mathbf{3}}$ & $\mathbf{t a}_{\mathbf{4}}$ & $\mathbf{t a}_{\mathbf{5}}$ & $\mathbf{L R}$ \\
\hline $\mathrm{p}_{1}$ & $680-720$ & $500-520$ & $920-1080$ & $490-515$ & $380-410$ & $310-320$ & $150-160$ & $1.84-1.08$ \\
\hline $\mathrm{p}_{2}$ & $630-650$ & $530-550$ & $340-365$ & $190-220$ & $120-135$ & $70-80$ & $60-70$ & $0.64-0.66$ \\
\hline $\mathrm{p}_{3}$ & $700-720$ & $710-725$ & $480-495$ & $250-260$ & $220-230$ & $110-115$ & $90-95$ & 0.68 \\
\hline
\end{tabular}

\section{Olecryptotendipes lenzi (Zorina)}

http://species-id.net/wiki/Olecryptotendipes_lenzi

Cryptotendipes lenzi Zorina, 2001: 31; Zorina, 2007: 350; Yan et al. 2005: 4

Specimens examined. China, Xinjiang Autonomous Region: $13 \hat{\jmath}$, Kaba Altay, Baihualin Nature Conservation area, 4803'39.05"N, 86²5'7.04"E, 15-16.vii.2002, Tang HQ, sweep net. 
Diagnostic characters. Based on Zorina (2001) and Yan et al. (2005), the species is easily distinguished from the other species of the genus by having shoulder-like posterior margin of tergite IX and digitiform superior volsella with a longitudinal keel.

Distribution. This species is distributed in the Russian Far East and northwestern China.

\section{Olecryptotendipes melasmus sp. n.}

urn:Isid:zoobank.org:act:A177BD2D-75F5-43F5-A269-E0FA3D9B22DC

http://species-id.net/wiki/Olecryptotendipes_melasmus

Figs $5-10$

Diagnosis. The species can be separated by the blackish brown spots on thorax and legs, the distally swollen anal point and the gonostylus with basal weak expansion.

Description. Male imago $(\mathrm{n}=2)$. Total length $3.13-3.60 \mathrm{~mm}$. Wing length $1.50-1.88$ $\mathrm{mm}$. Total length / wing length 1.91-2.09. Wing length / length of profemur 2.05-2.14.

Coloration. Thorax (Fig. 5) yellowish brown, with median black brown vittae. Femur of front leg yellowish green with distal parts dark brown, tibia dark brown except for median parts yellowish green, tarsi dark brown with basal $3 / 4$ of ta $_{1}$ yellowish green; femora and tibia of mid and hind legs yellowish green with distal parts of femora and basal parts of tibia dark brown, ta ${ }_{1}$ to ta ${ }_{5}$ lightly brown (Fig. 6). Abdomen yellowish green to brown, with tergite I-V yellowish green, tergite VI-VIII and hypopygium yellowish brown.

Head. AR 1.94-2.24. Ultimate flagellomere 620-760 mm long. Frontal tubercles absent. Temporal 13-15 setae, including 4-5 inner verticals, 4-6 outer verticals and 4-5 postorbitals. Clypeus with 13-21 setae. Tentorium 113-120 mm long, 27-37 mm wide. Palpomere lengths (in mm): 37-40, 39-45, 133-172, 133-158, 193-223. Palp segment $5^{\text {th }} / 3^{\text {rd }} 1.30-1.45$.

Thorax. Antepronotum with 2 setae, dorsocentrals $8-10$, acrostichals $4-7$, prealars 4-5. Scutellum with $12-15$ setae.

Wing (Fig. 7). VR 1.15-1.20, R with 12-16 setae, $\mathrm{R}_{1}$ with $11-16$ setae, $\mathrm{R}_{4+5}$ with 18-25 setae. Brachiolum 2 setae. Squama with 3-4 setae.

Legs. Front tibia with 3 subapical setae, 113-135, 133-150 and 145-170 $\mu \mathrm{m}$ long, spurs of middle tibia $28-30$ and 35-37 $\mu \mathrm{m}$ long excluding comb, comb with 22-34 teeth, $10-11 \mu \mathrm{m}$ long; spurs of hind tibia $30-32$ and $42-43 \mu \mathrm{m}$ long excluding comb, comb with 34-45 teeth, 10-12 $\mu \mathrm{m}$ long. Sensilla chaetica of mid and hind legs absent. Lengths (in $\mu \mathrm{m}$ ) and proportions of legs as in Table 2.

Table 2. Lengths $(\mu \mathrm{m})$ and proportion of legs of Olecryptotendipes melasmus sp. n., male $(\mathrm{n}=2)$.

\begin{tabular}{c|c|c|c|c|c|c|c|c}
\hline & $\mathbf{F e}$ & $\mathbf{T i}$ & $\mathbf{t a}_{\mathbf{1}}$ & $\mathbf{t a}_{\mathbf{2}}$ & $\mathbf{t a}_{\mathbf{3}}$ & $\mathbf{t a}_{\mathbf{4}}$ & $\mathbf{T a}_{\mathbf{5}}$ & $\mathbf{L R}$ \\
\hline $\mathrm{P}_{1}$ & $730-880$ & $500-650$ & $1100(1)$ & $580(1)$ & $430(1)$ & $350(1)$ & $150(1)$ & $1.69(1)$ \\
\hline $\mathrm{P}_{2}$ & $640-800$ & $530-700$ & $330-410$ & $180-220$ & $120-150$ & $70-85$ & 60 & $0.59-0.62$ \\
\hline $\mathrm{P}_{3}$ & $760-910$ & $720-940$ & $470-580$ & $280-340$ & $220-270$ & $120-140$ & $70-80$ & $0.62-0.65$ \\
\hline
\end{tabular}



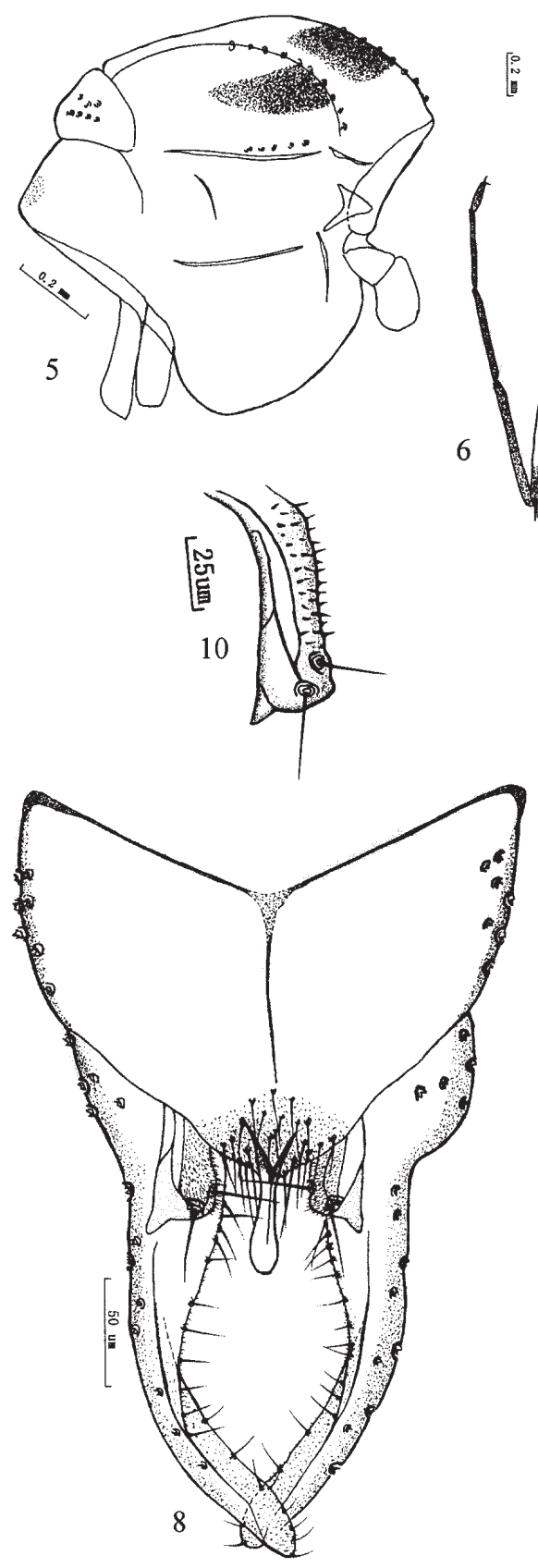

。

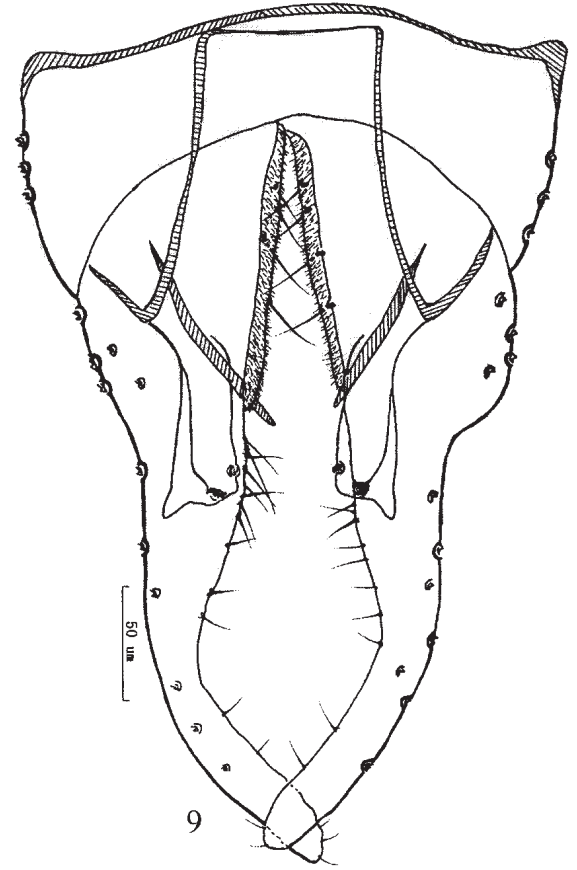

Figures 5-10. Olecryptotendipes melasmus sp. n., male. $\mathbf{5}$ thorax $\mathbf{6}$ leg $\mathbf{7}$ wing. 8 hypopygium (dorsal view) 9 hypopygium (ventral view) 10 superior volsella. 
Hypopygium (Figs 8-10). Tergite IX with weak shoulder-like corners, bearing 1320 setae at base of anal point. Laterosternite IX with 4-7 setae. Anal point originating from anterior of caudal margin of anal tergite in dorsal view, constricted basally, swollen apically, 43-50 mm long, 8-10 mm wide at base, $9-12 \mathrm{~mm}$ wide at apex. Anal tergite bands Y-shaped. Phallapodeme $68-80 \mathrm{~mm}$ long. Transverse sternapodeme 44-70 mm long. Superior volsella (Fig. 10) curved basally, straight distally, with large posterolateral projection, which is constricted medially forming a sharp angle, longitudinal membranous ridge present, bearing two long setae in distinct pits; covered with microtrichia in inner parts of superior volsella. Inferior volsella with reduced lobate caudal projection. Gonocoxite $85-100 \mathrm{~mm}$ long, with 4 strong inner marginal setae. Gonostylus 150-190 mm long, slightly swollen at base, concave medially, with rounded apex, bearing 8-20 setae along basal inner margin, and 10-14 setae along distal inner margin. HR 0.53-0.57; HV 1.89-2.09.

Type material. Holotype $\widehat{\overbrace{}}$ (BDN No. 04250). CHINA: Shaanxi Province, Baoji City, Feng County, Qinling, Dongyu, 33 $54^{\prime} 42.03^{\prime \prime N}, 106^{\circ} 31^{\prime} 21.10^{\prime \prime E}, 30$. vii. 1994, sweep net, W. Bu. Paratype (BDN No. 20598). CHINA: 10̄, Fujian Province, Jianning County, 26 $49^{\prime} 51.25^{\prime \prime N}, 116^{\circ} 50^{\prime} 45.90 " \mathrm{E}, 25$. ix. 2002, light trap, Z. Liu.

Etymology. From Greek, melasma, spot, in reference to the dark brown spots on the thorax and legs.

Distribution. The species is known from Palaearctic and Oriental China (Shaanxi Province; Fujian Province).

Systematic remarks. Male diagnosis: Based on the variation in Chinese material (O. exilis sp. n., O. melasmus sp. n.), the generic description given by Zorina (2007) should be emended as follows: "Antennal ratio 1.83-2.06" should be changed to "Antennal ratio 1.83-2.24". "Total length $3.0-3.5 \mathrm{~mm}$ " should be emended to "Total length $2.7-3.6 \mathrm{~mm}$."

Based on the description and figures of Zorina (2003), posterior margin of tergite IX of $O$. secundus not elongate but with caudolateral shoulders bearing 4 setae, which seem to $O$. exilis sp. n. The gonostylus of $O$. lenzi and $O$. secundus nearly parallelsided, but which is slender to apex of the two new species. The species $O$. secundus only with sclerotized superior volsella and without membranous ridges, it seems to $O$. exilis sp. n. with sclerotized beak-like protrusion. From the above, the generic characters of Olecryptotendipes should be emended as follows: "Posterior margin of tergite IX with caudolateral shoulders; gonostylus parallel-sided or slender to apex; superior volsella consisting of sclerotized part, dorsal and ventral setae present."

\section{Key to males of the genus Olecryptotendipes in the world}

1 Acrostichals absent; $\mathrm{R}$ and $\mathrm{R}_{1}$ without setae; superior volsella lacking microtrichia ventrally O. secundus (Zorina)

- $\quad$ Acrostichals present; $\mathrm{R}_{\text {and }} \mathrm{R}_{1}$ with setae; superior volsella with microtrichia ventrally 
2 Anal point swollen distally; thorax with dark brown spots ... O. melasmus sp. n. - $\quad$ Anal point parallel-sided, thorax without dark brown spots ......................... 3 3 Inferior volsella absent, gonostylus parallel-sided O. lenzi (Zorina) - Inferior volsella lobe-like, gonostylus swollen at base, moderately slender to apex O. exilis sp. n.

\section{Acknowledgements}

We want to thank Dr. M. Spies (Zoologische Staatssammlung München, Germany) and Prof. Ole Sxther (University of Bergen, Norway) for checking the specimens and providing lots of input on various levels of this work. Mr. Bingchun Ji and Mrs. Yufen Li made the slide preparations. Financial support received from the National Natural Science Foundation of China (NSFC) grants No. 31101653, 30870329 and Fauna of China (FY120100); the China Postdoctoral Science Foundation (20090226); Tianjin City High School Science \& Technology Fund Planning Project (20090608) and Tianjin Normal University Talent Introduction Foundation (5RL104) are thankfully acknowledged.

\section{References}

Sæther OA (1969) Some Nearctic Podonominae, Diamesinae and Orthocladiinae (Diptera: Chironomidae). Bulletin of the Fisheries Research Board of Canada 170: 1-154.

Sxther OA (1980) Glossary of chironomid morphology terminology (Diptera: Chironomidae). Entomologica Scandinavica Supplement 14: 1-51.

Sæther OA (1990) A review of the genus Limnophyes Eaton from the Holarctic and Afrotropical regions (Diptera: Chironomidae, Orthocladiinae). Entomologica Scandinavica Supplement 35: 1-139.

Yan CC, Tang, HQ, Wang XH (2005) A review of the genus Cryptotendipes Lenz (Diptera: Chironomidae) from China. Zootaxa 1086: 1-24. http://www.mapress.com/zootaxa/2005f/ z01086p024f.pdf

Zorina OV (2001) New species of the genera Cryptotendipes, Dicrotendipes, Microtendipes and Stenochironomus (Diptera, Chironomidae, Chironominae) from the Russian Far East. Vestnik Zoologii 35: 31-38.

Zorina OV (2003) Four new species of non-biting midges of the Harnischia complex (Diptera, Chironomidae, Chironominae) from Sakhalin Island (Russian Far East). Euroasian Entomological Journal 2: 221-225.

Zorina OV (2007) Olecryptotendipes, a new genus in the Harnischia complex (Diptera: Chironomidae) from the Russian Far East. In: Andersen T (Ed) Contributions to the Systematics and Ecology of Aquatic Diptera-A Tribute to Ole A. Sæther. The Caddis Press, Columbus, 347-351. 